\title{
A bacterial resolvase first exploits, then constrains intrinsic dynamics of the Holliday junction to direct recombination
}

\section{Sujay Ray ${ }^{1,2}$, Nibedita Pal ${ }^{1,2,3}$ and Nils G. Walter ${ }^{1 *}$}

${ }^{1}$ Single Molecule Analysis Group, Department of Chemistry, University of Michigan Ann Arbor, Michigan 48109, USA. ${ }^{2}$ These authors contributed equally: Sujay Ray; Nibedita Pal. ${ }^{3}$ Present address: Indian Institute of Science Education and Research (IISER) Tirupati, Tirupati 517507, Andhra Pradesh, India.*e-mail: nwalter@umich.edu 


\begin{abstract}
Homologous recombination forms and resolves an entangled DNA Holliday Junction (HJ)

critical for achieving genome repair. We use single-molecule observation and cluster analysis to probe how prototypic bacterial resolvase RuvC selects two of four possible HJ strands for cleavage. RuvC first exploits, then constrains intrinsic $\mathrm{HJ}$ isomer exchange and branch migration dynamics to direct cleavage toward only a desired, catalytically competent $\mathrm{HJ}$ conformation, thus controlling recombination products.
\end{abstract}


In Gram-negative bacteria such as Escherichia coli (E. coli), Holliday Junction (HJ) resolution is achieved by endonuclease RuvC, a well-studied, prototypical resolvase with extremely high topology and sequence specificity ${ }^{1-3}$. To achieve site-specific resolution, the $\mathrm{HJ}$ needs to be correctly positioned through branch migration of the junction, which occurs either spontaneously or catalyzed by the RuvAB complex ${ }^{4,5}$. A homodimer of RuvC can bind a HJ independently of $\operatorname{RuvAB}^{6-8}$ to distort the junction at the crossover point, eventually introducing two interdependent symmetric nicks via a two- $\mathrm{Mg}^{2+}$ catalytic mechanism ${ }^{2,9,10}$. Numerous in vitro studies with synthetic DNA have shown that RuvC recognizes a $\mathrm{HJ}$ structurally, but in sequence-independent manner. More specifically, in a recent study Zhou et al. ${ }^{11}$ showed that a RuvC-bound $\mathrm{HJ}$ retains its intrinsic fluctuations ${ }^{12-15}$ between two alternatively stacked, X-shaped conformational isomers (iso-I and iso-II), while an intermittent, multivalently bound, yet partially dissociated (PD) RuvC allows the HJ to undergo nearly unimpeded conformer exchange as well as branch migration ${ }^{11}$. However, it has remained unclear how RuvC achieves its remarkable cleavage specificity for the cognate $5^{\prime}$-ATT ${ }^{\downarrow} X-3^{\prime}$ sequence $\left(X=G / C\right.$; ${ }^{\downarrow}=$ cleavage site) to ensure proper control over chromosome segregation ${ }^{1,3,16-18}$.

Here we leverage single-molecule Förster resonance energy transfer (smFRET) to investigate the dynamic interaction of $E$. coli RuvC with a $\mathrm{HJ}$ that leads to sequence-specific junction resolution. Our results reveal that the resolvase binds to both cleavage-incompetent (iso-I) and -competent (iso-II) conformations, wherein the cognate sequences reside on the two bent and continuous (i.e., non-crossover) strands, respectively (Fig. 1a). Critically, RuvC takes advantage of the intrinsic $\mathrm{HJ}$ conformational and branch migration dynamics to find and then kinetically trap the catalytically active conformation through a (near-)irreversible switch from 
fluctuations between iso-I and a partially open state to fluctuations between iso-II and the partially open state. On the basis of these findings, we propose a model wherein RuvC exploits the intrinsic $\mathrm{HJ}$ dynamics until the cognate sequence enters the active site, inducing a snap-lock conformational switch that helps RuvC achieve high sequence specificity at little energetic cost.

To explore RuvC mediated cleavage, we chose an $\mathrm{HJ}$ sequence that contains the resolvase's cognate sequence within 5 base pairs of sequence homology and positioned for optimal cleavage activity ${ }^{16}$ across the junction on the $\mathrm{x}$ - and h-strands $\left(\mathrm{HJ}_{\mathrm{xh}}{ }^{\mathrm{R}}, \mathrm{Fig} .1 \mathrm{1a}\right)$. Cyanine-3 (Cy3, donor) and Cyanine-5 (Cy5, acceptor) dyes on two arms of the $\mathrm{HJ}$ allow monitoring the conformational dynamics using total internal reflection fluorescence (TIRF) based smFRET (Fig.

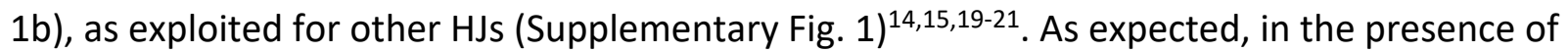
divalent metal ions $\mathrm{Mg}^{2+}$ or $\mathrm{Ca}^{2+} \mathrm{HJ}_{x h}{ }^{\mathrm{R}}$ undergoes fast dynamic transitions between stacked isomeric conformers ${ }^{14,15,19-21}$ iso-I and iso-Il of high $\left(\mathrm{E}_{\mathrm{FRET}}=0.65 \pm 0.12\right)$ and low $\left(\mathrm{E}_{\mathrm{FRET}}=\right.$ $0.21 \pm 0.11)$ FRET efficiencies, respectively (Fig. 1c). The interconversion rate constants of $k_{l \rightarrow l l}=$ $2.8 \pm 0.3 \mathrm{~s}^{-1}$ and $\mathrm{k}_{/ / \rightarrow l}=4.0 \pm 0.4 \mathrm{~s}^{-1}$ at $5 \mathrm{mM} \mathrm{Mg}^{2+}$ decrease gradually with increasing $\mathrm{Mg}^{2+}$ concentration (Supplementary Table 1; Supplementary Fig. 2). Occasional branch migration of the $\mathrm{HJ}$ was detected as a variation in FRET dynamics by visual inspection, as observed before ${ }^{11}$. A Fano-factor of $>1$, evaluating non-randomness through the variability in number of pairwise transitions relative to its mean ${ }^{22,23}$, was found to be a signature of the co-existence of regimes of slow and fast FRET transitions representing iso- $/ \leftrightarrow$ iso- $/ /$ isomerization and branch migration, respectively (Fig. 1c and Supplementary Fig. 3). When $\mathrm{Mg}^{2+}$ was replaced with $\mathrm{Ca}^{2+}$, only slightly slower kinetics were observed, indicating that $\mathrm{HJ}_{\mathrm{xh}}{ }^{\mathrm{R}}$ shows similar two-state isomerization 
dynamics in $\mathrm{Mg}^{2+}$ or $\mathrm{Ca}^{2+}$ as other HJs (Supplementary Fig. 1$)^{14,15,19-21}$, with a slight (60\%:40\%) iso-I:iso-/l bias dictated largely by the junction sequence ${ }^{19,24}$.

The $\mathrm{HJ}_{\mathrm{xh}}{ }^{\mathrm{R}}$ cleavage activity of RuvC was tested upon incubation for $1 \mathrm{~h}$ at $37^{\circ} \mathrm{C}$ in standard cleavage buffer (20 mM Tris- $\mathrm{HCl}$, pH-8, 20 mM NaCl, 1 mM EDTA, 1mM DTT, 0.1mg/ml BSA, 10 $\mathrm{mM} \mathrm{MgCl}$ ) by monitoring the appearance of the shortened Cy5 labeled cleavage product using denaturing gel electrophoresis (Fig. 1d). Under these conditions, increasing the RuvC concentration yielded an isotherm with a half-saturation value $K_{1 / 2}$ of $\sim 66 \mathrm{nM}$ RuvC (Supplementary Fig. 4). In our TIRF microscope, we instead monitored the disappearance of the no longer surface-coupled Cy3-labeled product spots (Fig. 1e), confirming that RuvC resolves single $\mathrm{HJ}_{\mathrm{xh}}{ }^{\mathrm{R}}$ molecules at $37^{\circ} \mathrm{C}$ in standard cleavage buffer. At a saturating concentration of RuvC (400 nM), the cleavage rate constant was $1.58 \times 10^{-3} \mathrm{~s}^{-1}$, similar to previous studies ${ }^{16}$, and became zero when $\mathrm{Mg}^{2+}$ was replaced with $\mathrm{Ca}^{2+}$ (Fig. 1f). Bulk cleavage reactions performed at room temperature and/or only $5 \mathrm{mM} \mathrm{Mg}^{2+}$ showed significantly reduced cleavage (Fig. 1f), as expected ${ }^{25}$, providing an opportunity to decouple the impact of RuvC on $\mathrm{HJ}_{\mathrm{xh}}{ }^{\mathrm{R}}$ conformational dynamics observed by smFRET from HJ cleavage (which is 35 -fold less efficient at our standard decoupled smFRET conditions of $20^{\circ} \mathrm{C}$ in $5 \mathrm{mM} \mathrm{Mg}^{2+}$ compared to $37^{\circ} \mathrm{C}$ in $10 \mathrm{mM} \mathrm{Mg}^{2+}$, Fig. 1f).

Using decoupled smFRET conditions in the presence of saturating (400 nM) RuvC, we observed that the dynamic exchange between iso-I and iso-I/ becomes more complex (compare Figs. $2 a$ and $2 b)$; in particular, a new mid-FRET state appears $\left(E_{F R E T}=0.4 \pm 0.1\right)$ that lies between the high-FRET iso-I $\left(\mathrm{E}_{\mathrm{FRET}}=0.64 \pm 0.13\right)$ and the low-FRET iso-II $\left(\mathrm{E}_{\mathrm{FRET}}=0.17 \pm 0.1\right)$. This state is similar to the planar open conformation of the $\mathrm{HJ}$ in the absence of divalent metal ions ${ }^{10,11}$, and is henceforth termed "open", or Op. Upon replacing $\mathrm{Mg}^{2+}$ with $\mathrm{Ca}^{2+}$, this Op state disappears, 
leaving only iso-I $\left(\mathrm{E}_{\mathrm{FRET}}=0.68 \pm 0.09\right)$ and iso-II $\left(\mathrm{E}_{\mathrm{FRET}}=0.15 \pm 0.08\right.$, Fig. $\left.2 \mathrm{c}, \mathrm{d}\right)$. The observation of Op only in the presence of RuvC and $\mathrm{Mg}^{2+}$, which are both required for cleavage, suggests that this state is relevant for $\mathrm{HJ}$ resolution. In fact, visual inspection revealed that $\sim 40 \%$ of smFRET trajectories visit Op, while the remainder continues to show two-state behavior only, most likely due to limited RuvC binding ${ }^{16}$ (Fig. 2e). Notably, a similar fraction ( $37 \%$ ) of HJ molecules is cleaved under optimal cleavage conditions (Fig. 1f), further supporting the notion that the population accessing the Op state under decoupled smFRET conditions is RuvC-bound and cleavage-enabled.

To classify the dynamic features of these RuvC-HJ complexes, we utilized modelindependent hierarchical Single Molecule Cluster Analysis (SiMCAn) ${ }^{26,27}$ of N=673 Hidden Markov Model (HMM) idealized smFRET traces from four experimental conditions (Fig. 2a-d): 5 $\mathrm{mM} \mathrm{Mg}^{2+}, 5 \mathrm{mM} \mathrm{Mg}^{2+}$ plus $400 \mathrm{nM}$ RuvC, $5 \mathrm{mM} \mathrm{Ca}^{2+}$, and $5 \mathrm{mM} \mathrm{Ca}^{2+}$ plus $400 \mathrm{nM}$ RuvC. Using a hierarchical cluster tree, we grouped traces with similar FRET states and kinetics into four clusters. We observed that $43 \%$ of the traces from the $\mathrm{Mg}^{2+} / \mathrm{RuvC}$ condition show distinct dynamic features, suggesting that they represent the $\sim 40 \%$ RuvC-bound, cleavage-enabled molecules; we therefore termed this cluster " $R$ ". By contrast, the remaining clusters, termed S1, $\mathrm{S} 2$, and S3, showed protein-free, salt-condition-like iso- $/ \leftrightarrow$ iso-II dynamics and dominate in the absence of RuvC and/or presence of $\mathrm{Ca}^{2+}$ (Fig. 2f, g; Supplementary Fig. 5). Notably, a full 84\% of all traces in the R-cluster arise from the $\mathrm{Mg}^{2+} /$ RuvC condition, further supporting a unique behavior associated with the binding of both $\mathrm{Mg}^{2+}$ and RuvC. Accordingly, the FRET probability distribution of R-cluster traces populates the three conformations iso-I (high-FRET), Op (midFRET) and iso-II (low-FRET), with enrichment of Op from $23 \%$ to $50 \%$ unique to the $\mathrm{Mg}^{2+} / \mathrm{RuvC}^{2}$ 
condition (compare Figs. $2 \mathrm{~h}$ and $2 \mathrm{~b}$, Supplementary Fig. 5c-d). The existence of iso- $I$ and iso- $/ /$ in these traces suggests that both conformers can be bound by RuvC in sequence and isoformindependent manner, and with unperturbed interconversion dynamics, consistent with prior observations of the PD state of RuvC ${ }^{11}$.

A transition occupancy density plot (TODP, Fig. 2i, Supplementary Fig. 5e) generated from over 900 transitions of RuvC bound R-cluster traces revealed three rapid, reversible conformational transitions: iso- $I_{R} \leftrightarrow$ iso-I $I_{R}$ (low $\leftrightarrow$ high-FRET; $21 \%$ ), iso-II $\leftrightarrow O p_{R}$ (low $\leftrightarrow$ midFRET; $57 \%$ ), and iso- $I_{R} \leftrightarrow O p_{R}$ (high $\leftrightarrow$ mid-FRET; $22 \%$ ). Among individual traces featuring the cleavage-enabled Op state, we observed three different classes: with only pairwise iso- $I I_{R} \leftrightarrow O p_{R}$ transitions (45\%), with only pairwise iso- $I_{R} \leftrightarrow O p_{R}$ transitions (10\%), and with a slow interconversion from one to the other pairwise transition behaviors (35\% "mixed" traces; Fig. 2e, Supplementary Fig.6). To further probe this interconversion, we segmented the mixed traces into their early and late phases and performed a second layer of SiMCAn. As expected, the early and late trace segments belong to distinct clusters (Supplementary Fig. 7a), for which TODPs revealed that the early and late phases preferably show iso- $I_{R} \leftrightarrow O p_{R}$ and iso- $I I_{R} \leftrightarrow O p_{R}$ behavior, respectively (Fig. $2 \mathrm{j}$ ). That is, we observe a strong propensity (>90\% probability) of the mixed traces to start from iso- $I_{R} \leftrightarrow O p_{R}$ behavior and end with iso- $I_{R} \leftrightarrow O p_{R}$ behavior (Fig. $2 \mathrm{k}$ ). Consistent with this finding, we observe an increase in traces showing the iso- $I_{R} \leftrightarrow O p_{R}$ to iso$I_{R} \leftrightarrow O p_{R}$ interconversion from $20 \%$ to $32 \%$ when collecting traces at later time points (Supplementary Fig.7b). Significantly, Fano factor calculations reveal that, while the early iso$I_{R} \leftrightarrow O p_{R}$ transitions are non-randomly (non-Poisson) distributed, the late-phase iso- $I_{R} \leftrightarrow O p_{R}$ 
transitions are random (Fig. 2l), providing direct evidence that the early phase still allows for PD-state mediated HJ branch migration, whereas the late phase does not.

To contextualize the complex $\mathrm{HJ}$ conformational isomerization we discovered upon RuvC binding under cleavage-enabled conditions, it is instructive to inspect the available crystal structures of the RuvC-HJ complex (Fig. 3). More than twelve amino acids from each monomer of RuvC form redundant contacts with the junction ${ }^{6}$, allowing the resolvase to remain loosely associated in the PD state so that the $\mathrm{HJ}$ can transition between isomers iso-I and iso-II as well as branch migrate (Fig. 3) ${ }^{11}$. Once the cognate cleavage sequence is approaching the enzyme's catalytic core near the junction via this rapid sampling, charged amino acids such as Arg76 in the core of RuvC stabilize the G-C base of the consensus cleavage sequence via amino acidnucleo base cation-pi interactions ${ }^{6}$. This positions the thymine residues of the cleavage sequence in hydrophobic pockets of the catalytic sites ${ }^{3,8}$ for $\mathrm{HJ}$ resolution. We propose that, once the cleavage-competent iso-I/ conformation is recognized, it snap-locks into place as evidenced by the near-irreversible switch from the early iso- $I_{R} \leftrightarrow O p_{R}$ to the late iso- $I I_{R} \leftrightarrow O p_{R}$ pairwise transition behavior, positioning all RuvC-HJ contacts for catalysis (Fig. 3). At this stage, both transitions to iso-I and branch migration are inhibited, while the cleavage-competent iso-II conformation is snap-locked, ready for $\mathrm{Mg}^{2+}$-mediated catalysis. This model explains how the prototypic bacterial resolvase RuvC selects two of four possible $\mathrm{HJ}$ strands for cleavage. The RuvC dimer first recognizes and binds a $\mathrm{HJ}$ purely topologically, independent of sequence and conformational isomerization, then distorts the $\mathrm{HJ}$ to a partially open (Op) form ${ }^{10}$ while allowing it to remain dynamic by adopting its PD state. RuvC thus can utilize the intrinsic HJ dynamics through rapid conformational sampling and branch migration until it detects the 
cognate sequence in the continuous strand (here, of isomer iso-II), upon which amino acidnucleo base interactions act as constraints to stabilize a cleavage-competent conformation through a near-irreversible conformational switch to iso- $I_{R} \leftrightarrow O p_{R}$, disallowing the cleavageincompetent iso-I at minimal energetic cost. This constrained, snap-locked state is the basis for specificity during RuvC mediated cleavage, leading to a targeted set of desired recombination end products.

In conclusion, we here have revealed the basis of RuvC's unique combination of topological and sequence specificity, which may open exciting possibilities for antimicrobial therapy ${ }^{28,29}$ since sequence selectivity distinguishes RuvC from many eukaryotic/mammalian HJ resolvases ${ }^{30-34}$. Additionally, we anticipate that our quantitative single molecule cluster analysis will serve as a powerful tool to unravel the differentiating mechanisms of other DNA binding and processing enzymes.

\section{Methods}

Methods, including statements of data availability and any associated accession codes and references, are available at ...

\section{References}

1. Shah, R., Bennett, R.J. \& West, S.C. Cell 79, 853-864 (1994).

2. $\quad$ Bennett, R.J. \& West, S.C. Proc. Natl. Acad. Sci. USA 92, 5635-5639 (1995).

3. Shida, T., Iwasaki, H., Saito, A., Kyogoku, Y. \& Shinagawa, H. J. Biol. Chem. 271, 26105-26109 (1996).

4. Yamada, K., Ariyoshi, M. \& Morikawa, K. Curr. Opin. Struct. Biol. 14, 130-137 (2004).

5. Michel, B., Sinha, A.K. \& Leach, D.R.F. Microbiol. Mol. Biol. Rev. 82(2018).

6. Gorecka, K.M., Komorowska, W. \& Nowotny, M. Nucleic Acids Res. 41, 9945-9955 (2013).

7. Chen, L., Shi, K., Yin, Z. \& Aihara, H. Nucleic Acids Res. 41, 648-656 (2013).

8. Ariyoshi, M. et al. Cell 78, 1063-1072 (1994).

9. $\quad$ Bennett, R.J., Dunderdale, H.J. \& West, S.C. Cell 74, 1021-1031 (1993).

10. Bennett, R.J. \& West, S.C. J. Mol. Biol. 252, 213-226 (1995).

11. Zhou, R. et al. Nat. Chem. Biol. 15, 269-275 (2019). 
12. Nowakowski, J., Shim, P.J., Prasad, G.S., Stout, C.D. \& Joyce, G.F. Nat. Struct. Biol. 6, 151-156 (1999).

13. Eichman, B.F., Vargason, J.M., Mooers, B.H. \& Ho, P.S. Proc. Natl. Acad. Sci. USA 97, 3971-3976 (2000).

14. McKinney, S.A., Declais, A.C., Lilley, D.M. \& Ha, T. Nat. Struct. Biol. 10, 93-97 (2003).

15. Joo, C., McKinney, S.A., Lilley, D.M. \& Ha, T. J. Mol. Biol. 341, 739-751 (2004).

16. Bennett, R.J. \& West, S.C. Proc. Natl. Acad. Sci. USA 93, 12217-12222 (1996).

17. White, M.F., Giraud-Panis, M.J., Pohler, J.R. \& Lilley, D.M. J. Mol. Biol. 269, 647-664 (1997).

18. Fogg, J.M., Schofield, M.J., White, M.F. \& Lilley, D.M. Biochemistry 38, 11349-11358 (1999).

19. McKinney, S.A., Freeman, A.D., Lilley, D.M. \& Ha, T. Proc. Natl. Acad. Sci. USA 102, 5715-5720 (2005).

20. Hohng, S. et al. Science 318, 279-283 (2007).

21. Tang, J., Sun, Y., Pang, S. \& Han, K.Y. Sci. Rep. 7, 10945 (2017).

22. Rinaldi, A.J., Lund, P.E., Blanco, M.R. \& Walter, N.G. Nat Commun 7, 8976 (2016).

23. Eden, U.T. \& Kramer, M.A. J. Neurosci. Methods 190, 149-152 (2010).

24. Miick, S.M., Fee, R.S., Millar, D.P. \& Chazin, W.J. Proc. Natl. Acad. Sci. USA 94, 9080-9084 (1997).

25. Takahagi, M., Iwasaki, H. \& Shinagawa, H. J. Biol. Chem. 269, 15132-15139 (1994).

26. Blanco, M.R. et al. Nat. Methods 12, 1077-1084 (2015).

27. Rohlman, C.E., Blanco, M.R. \& Walter, N.G. Methods Enzymol. 581, 257-283 (2016).

28. Loughlin, M.F., Barnard, F.M., Jenkins, D., Sharples, G.J. \& Jenks, P.J. Infect. Immun. 71, 20222031 (2003).

29. Garcia, A.D., Otero, J., Lebowitz, J., Schuck, P. \& Moss, B. J. Biol. Chem. 281, 11618-11626 (2006).

30. Wyatt, H.D., Sarbajna, S., Matos, J. \& West, S.C. Mol. Cell 52, 234-247 (2013).

31. Ciccia, A., Constantinou, A. \& West, S.C. J. Biol. Chem. 278, 25172-25178 (2003).

32. Chen, X.B. et al. Mol. Cell 8, 1117-1127 (2001).

33. Ip, S.C. et al. Nature 456, 357-361 (2008).

34. Sobhy, M.A. et al. Nucleic Acids Res. 47, 1935-1949 (2018).

\section{Acknowledgements}

This work was supported by a NSF Award DMR-1607854 (to S.G.-T.) and NIH grant 2R01 GM062357 (to N.G.W.). We thank Dr. Alexander Johnson-Buck for careful reading of the manuscript and thoughtful suggestions.

\section{Author contributions}

S.R., N.P., and N.G.W. designed the study and wrote the manuscript. 
S.R. and N.P. performed all experiments and analyzed the data

S.R. and N.P. contributed equally.

\section{Competing interests}

The authors declare no competing interests.

\section{Additional information}

Supplementary information is available for this paper at ...

Reprints and permissions information is available at www.nature.com/reprints.

Correspondence and requests for materials should be addressed to N.G.W. 
Ray, Pal \& Walter, Figure 1

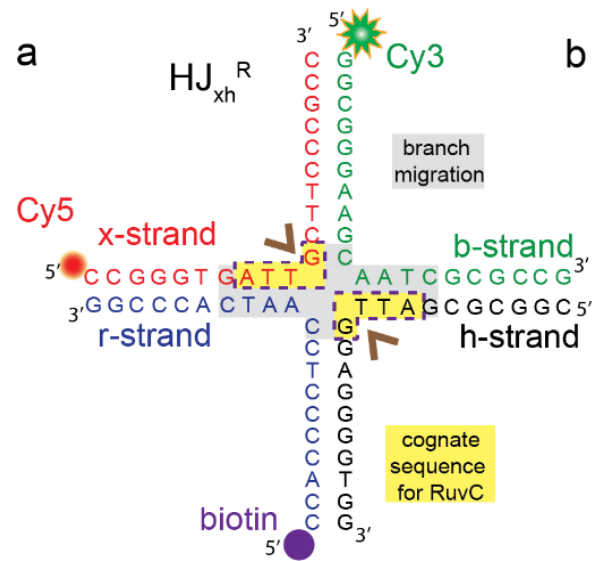

d

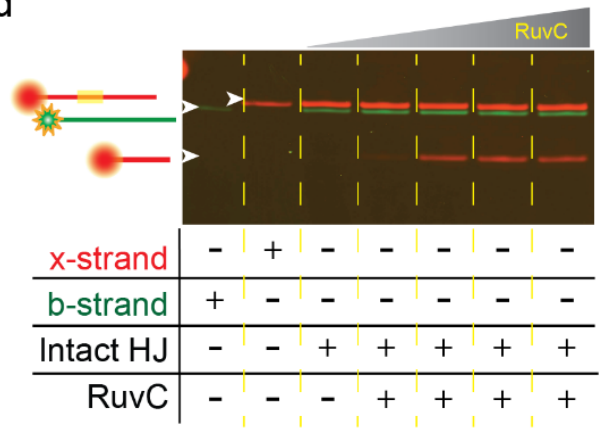

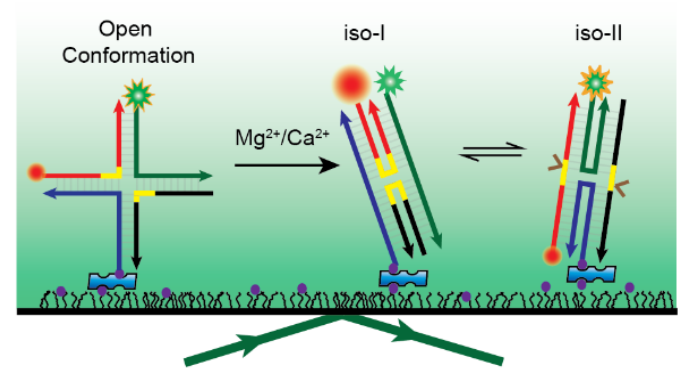

e

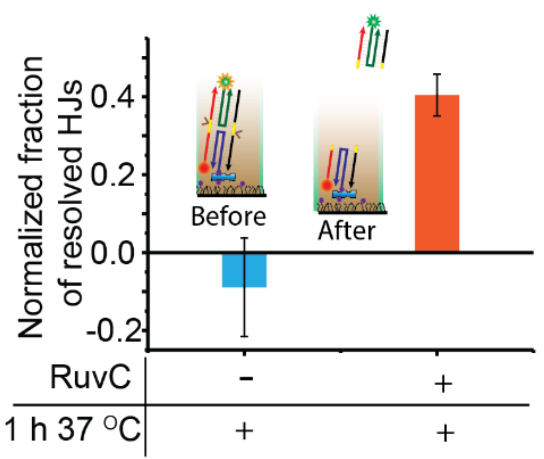

C branch

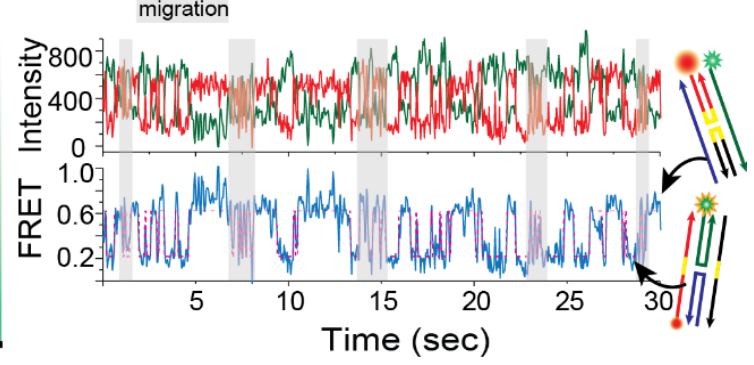

f

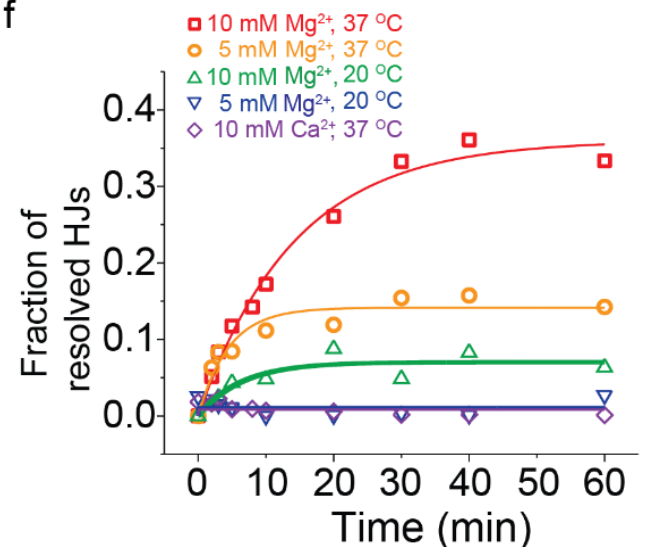

Figure 1 | Holliday junction assay design and cleavage by RuvC. a, Schematic and sequence of the $\mathrm{HJ}_{\mathrm{xh}}{ }^{\mathrm{R}}$ design. The nucleotides highlighted in yellow represent the cognate cleavage sequence for RuvC. The nucleotides highlighted in gray represent the region of the junction that can potentially branch migrate due to sequence homology. The brown arrows indicate the cleavage sites. The 5' ends of the $r, x$, and $b$-strand are labeled with biotin, Cy 5 and Cy3, respectively. $\mathbf{b}$, Schematic of our smFRET assay under TIRF illumination to monitor the dynamics of $\mathrm{HJ}_{\mathrm{xh}}{ }^{\mathrm{R}}$ molecules. The $\mathrm{HJ}$ transitions between two isomeric states iso-l and iso-II. The cognate sequences are shown in yellow in the bent, cleavage-incompetent iso-I and continuous, cleavage-competent iso-I/ strands. c, Representative single-molecule time trajectory showing donor (green) and acceptor (red) intensity in the top panel, FRET (blue) and HMM fitting (magenta) in the bottom panel. Fast transitions highlighted in gray are attributed to branch migration dynamics. Representative cartoons indicate the conformational states associated with particular FRET values. d, Superimposed Cy3 (green) and 
Cy5 (red) scans of a denaturing urea-polyacrylamide gel showing RuvC-mediated cleavage of $\mathrm{HJ}_{x h}{ }^{\mathrm{R}}$ in the presence of varying concentrations of RuvC. The top bands represent the full-length 20-nt-long DNA; the bottom bands are the cleaved 10-nt-long DNA product. The schematics on the right-hand side represent the relative length of DNA corresponding to each band. The Cy3-labeled $b$ strand is not cleaved due to the absence of a cleavage sequence. e, Quantification of cleavage using a single molecule assay.

Normalized ratios of number of colocalized Cy3-Cy5 spots and number of all Cy5 spots before and after cleavage are plotted for the presence and absence of RuvC. Without RuvC, this ratio remains close to zero. In the presence of $10 \mathrm{mM} \mathrm{Mg}^{2+}$ and $400 \mathrm{nM}$ RuvC incubated at $37^{\circ} \mathrm{C}$ for $1 \mathrm{~h}$, this ratio becomes $0.40 \pm 0.05$, representing RuvC-mediated cleavage. The inset shows a schematic of the experiment before and after cleavage in the presence of RuvC. $\mathbf{f}$, Quantitative analysis of a time course of RuvC-mediated cleavage of $\mathrm{HJ}_{\mathrm{xh}}{ }^{\mathrm{R}}$ in the presence of $10 \mathrm{mM} \mathrm{Mg}^{2+}$ at $37^{\circ} \mathrm{C}$ (red square), $5 \mathrm{mM} \mathrm{Mg}^{2+}$ at $37^{\circ} \mathrm{C}$ (orange circle), $10 \mathrm{mM} \mathrm{Mg}^{2+}$ at $20{ }^{\circ} \mathrm{C}$ (green up triangle), $5 \mathrm{mM} \mathrm{Mg}^{2+}$ at $20^{\circ} \mathrm{C}$ (blue down triangle), and $10 \mathrm{mM} \mathrm{Ca}^{2+}$ at $37^{\circ} \mathrm{C}$ (purple diamond) at $400 \mathrm{nM}$ RuvC. Saturation curves are fit to the data. The fraction of $\mathrm{HJ}_{\mathrm{xh}}{ }^{\mathrm{R}}$ molecules cleaved by RuvC decreases with decreasing temperature and decreasing $\mathrm{Mg}^{2+}$ concentration. This provides us with an opportunity to decouple conformational dynamics from cleavage. 
Ray, Pal \& Walter, Figure 2
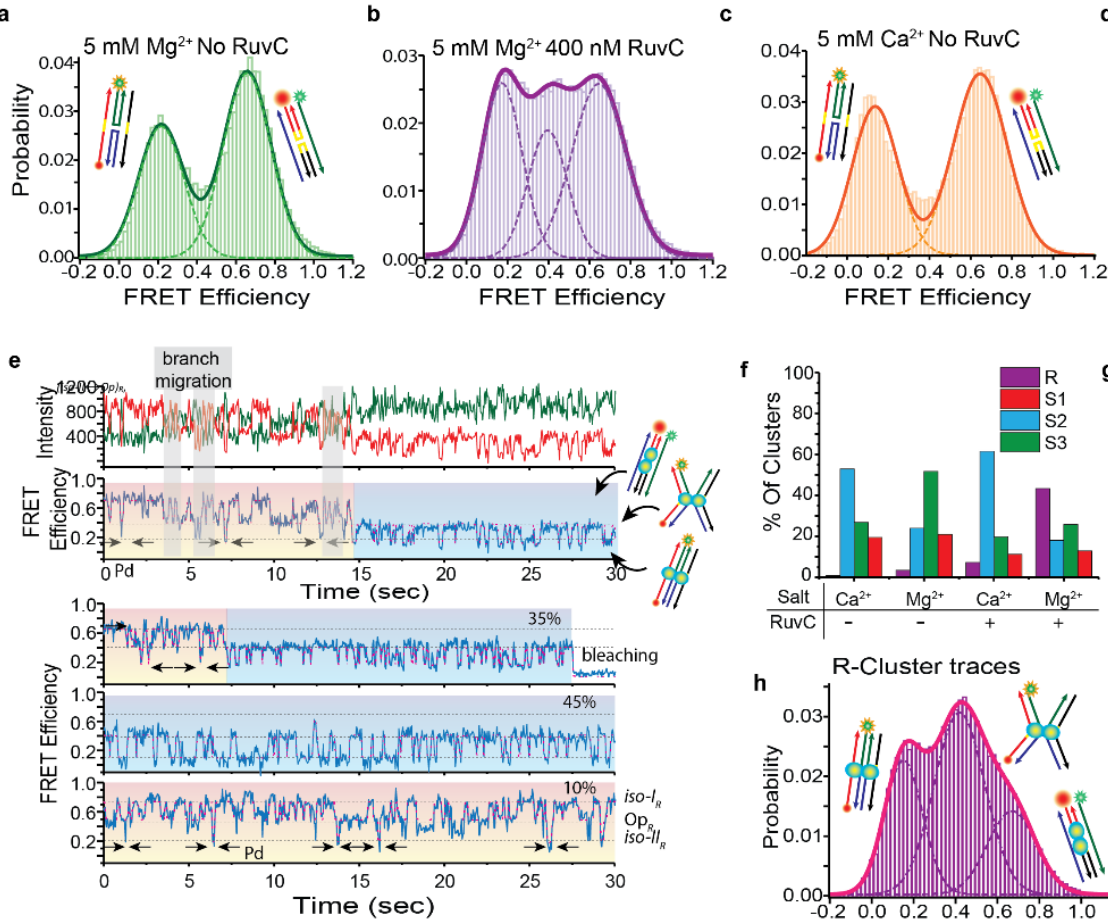

FRET Efficiency
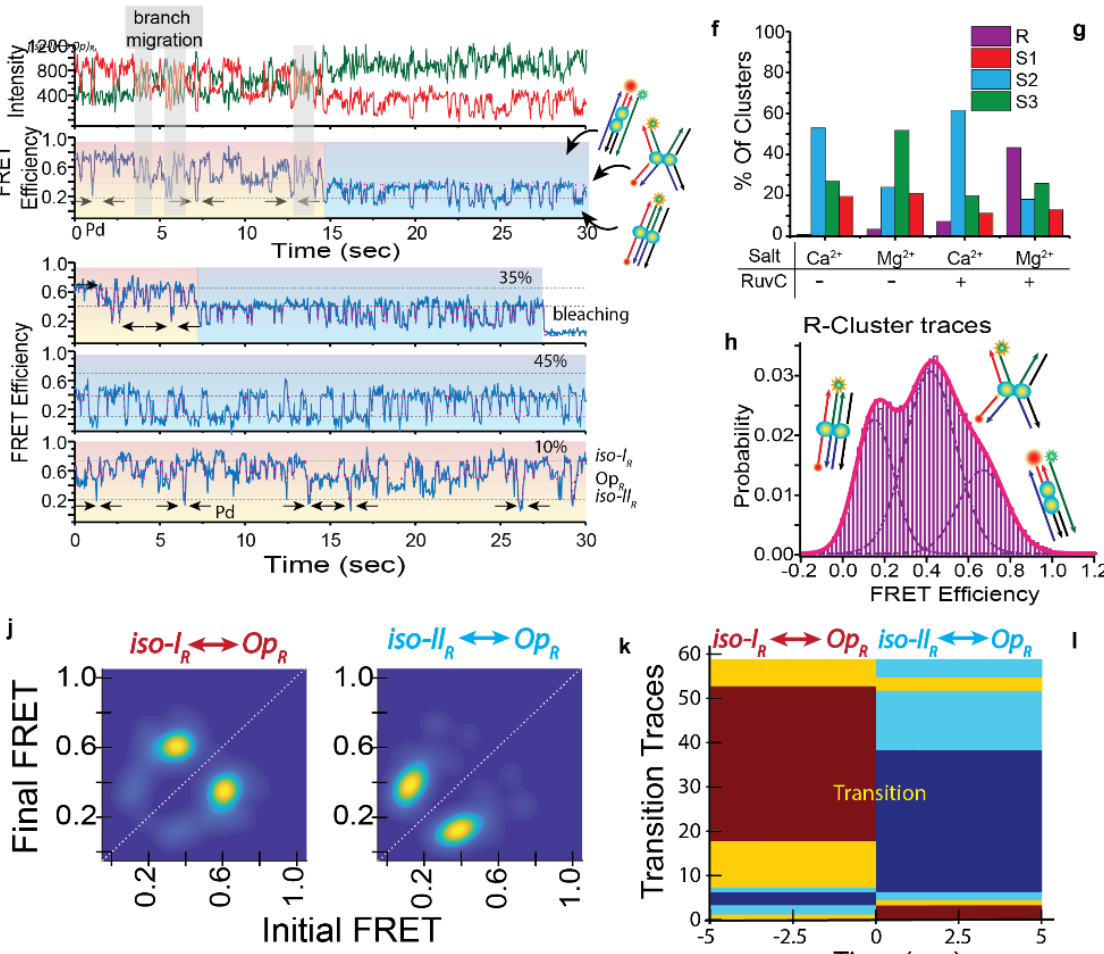

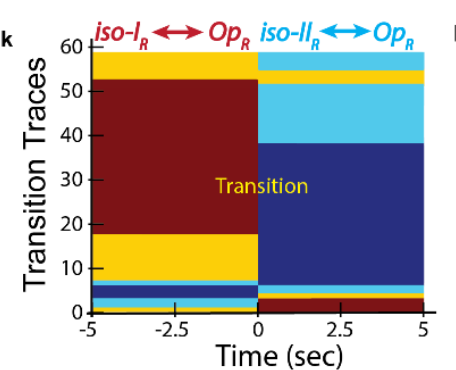

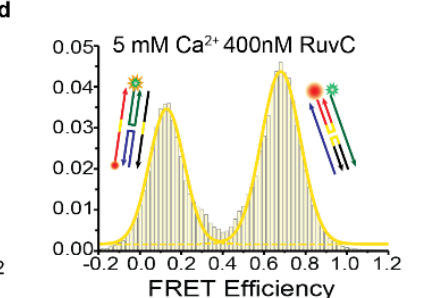
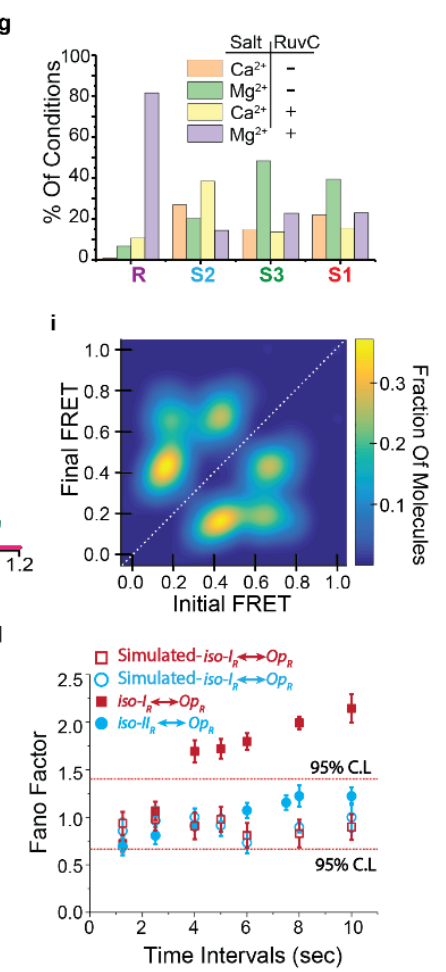

Figure 2 | Single molecule Cluster Analysis (SiMCAn) of smFRET trajectories reveals dynamic behaviors unique to the cleavagecompetent HJ-RuvC complex. FRET probability distributions of a, $5 \mathrm{mM} \mathrm{Mg}^{2+}$ without RuvC, b, $5 \mathrm{mM} \mathrm{Mg}^{2+}$ plus $400 \mathrm{nM}$ RuvC c, $5 \mathrm{mM}$ $\mathrm{Ca}^{2+}$ without RuvC, and d, $5 \mathrm{mM} \mathrm{Ca}{ }^{2+}$ plus 400nM RuvC. Multi-peak Gaussian functions are used to fit each histogram. Associated 
structural cartoons depict the conformational states corresponding to each FRET population. e, Representative fluorescence intensity trace of Cy3 (green) and Cy5 (red) fluorophore, FRET efficiency (blue) and HMM fitting (magenta) from the $5 \mathrm{mM} \mathrm{Mg}^{2+} \mathrm{plus}$ 400nM RuvC condition (top panel). Three additional FRET efficiency traces show different types of behaviors under the same conditions (bottom panel), with percentages delineating the fractions of traces displaying such behavior. Associated cartoons represent most likely conformations corresponding to each FRET value. The orange background represents iso-I $I_{R} \leftrightarrow O p_{R}$ behavior and the blue background represents iso- $I_{R} \leftrightarrow O p_{R}$ behavior. Sudden visits to non-prevalent FRET states, assigned as RuvC's PD state (marked by black arrows) and branch migration (marked by gray background), are observed in the iso- $I_{R} \leftrightarrow O p_{R}$ behavior and absent from the iso- $I I_{R} \leftrightarrow O p_{R}$ behavior. $f$, Summary of SiMCAn results, showing bar graphs with occupancy of the four clusters found in four experimental conditions. Clusters S1, S2 and S3 are distributed over all different conditions. By contrast, the R-cluster is predominantly found in the $5 \mathrm{mM} \mathrm{Mg}^{2+}$ plus $400 \mathrm{nM} \mathrm{RuvC}$ condition, indicating that the R-cluster is associated with the RuvC- $\mathrm{HJ}_{\mathrm{xh}}{ }^{\mathrm{R}}$ complex. g, Bar graph showing the fraction of molecules from of each experimental condition contributing to the four clusters. More than $80 \%$ of the molecules in the R-cluster belong to the condition where both $\mathrm{Mg}^{2+}$ and RuvC are present. $\mathbf{h}$, FRET efficiency histogram calculated from the traces belonging to the R-cluster, shown with multi-peak Gaussian fits. Associated cartoons represent different RuvC-HJ complex conformations corresponding to different FRET states. i, Transition Occupancy Density Plots (TODPs) from R-cluster traces showing the fraction of HJs that undergo conformational transitions from a given initial FRET state to a specific final FRET. Three main sets of bidirectional transitions are observed, representing iso- $I I_{R} \leftrightarrow O p_{R}$, iso- $I_{R} \leftrightarrow$ iso- $I I_{R}$, and iso- $I_{R} \leftrightarrow O p_{R}$ behavior, respectively. j, TODP calculated from the early phases of the traces (red/yellow) show only (iso-I $\leftrightarrow$ Op) $)_{R}$ behavior (left panel), whereas segments from the later phases of the traces (cyan/blue) show iso- $I_{R} \leftrightarrow O p_{R}$ behavior (right panel). $\mathbf{k}$, The early and late phases of the mixed R-cluster traces were classified into four clusters (annotated in red, yellow, cyan and blue) using SiMCAn. The early phases, representing iso- $I_{R} \leftrightarrow O p_{R}$ behavior, and the late phases, representing iso- $I I_{R} \leftrightarrow O p_{R}$ behavior, belongs to different clusters (see Supplementary Fig.7a). Upon reconstitution of their early and late phases with their clusters annotated, a clear propensity becomes evident for individual traces to converge over time onto the iso- $I I_{R} \leftrightarrow O p_{R}$ behavior. I, The Fano factor was calculated across various time intervals for the iso- $I_{R} \leftrightarrow O p_{R}$ behavior (solid red square), iso- $I_{R} \leftrightarrow O p_{R}$ behavior (solid blue circle) and simulated Poisson data same in length and number of traces for the same behaviors (open red square, open blue circle) respectively. The dashed lines indicate the $95 \%$ confidence level of the data. The iso- $I I_{R} \leftrightarrow O p_{R}$ behavior Fano factor values deviate from 1, indicating a non-random underlying distribution, while the iso-II-loaded behavior Fano factor data remain close to 1 , indicating a Poisson distribution. 
Ray, Pal \& Walter, Figure 3
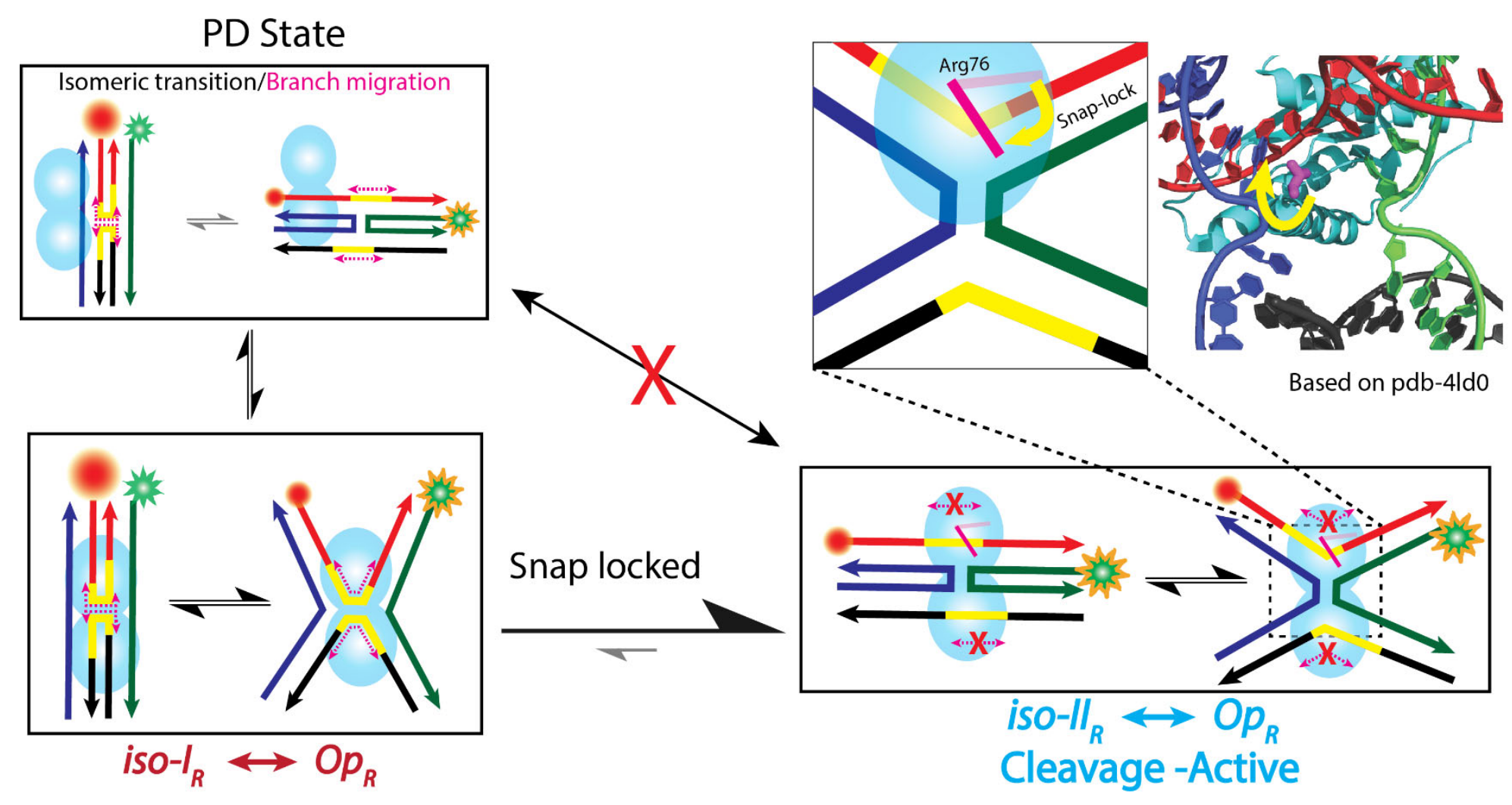

Figure 3 | Proposed model for RuvC-mediated, site-specific Holliday junction resolution. When RuvC binds to iso-I, it forms an Open state $(\mathrm{Op})$ where the cleavage sequence forms a shallow angle. RuvC allows the $\mathrm{HJ}$ junction to remain dynamic by partially dissociating from it (PD state). Isomeric transitions and branch migration are both allowed at this stage. Once a suitable cognate sequence is recognized, a (near-)irreversible transition leads to snap-locking of iso- $/ /$ into the active site of the resolvase via amino acid-nucleo base interactions such as through Arg76, wherein the cleavage site can only still adopt the wider angle of a now cleavage-active Op state. Other conformational transitions such as to the PD state are suppressed. 